\title{
Endovenous Radiofrequency Ablation of Superficial and Perforator Veins
}

\author{
Steven M. Roth, MD, MS ${ }^{\mathrm{a}, \mathrm{b}, *}$ \\ ${ }^{\text {a }}$ Vein Care Pavilion of the South, 447 North Belair Road, Suite 103, Evans, GA 30809, USA \\ ${ }^{\mathrm{b}}$ University Surgical Associates, 818 St. Sebastian Way, Augusta, GA 30901, USA
}

In general, the overall lack of enthusiasm for the treatment of chronic venous insufficiency in the past stemmed from less objective testing methods and more invasive surgical procedures that were fraught with increased patient morbidity and dissatisfaction. Primary venous insufficiency and varicose veins are important vascular conditions that affect 25 million people in Western civilization [1]. The management of superficial and perforator venous reflux disease has progressed to encompass more objective diagnostic methods, including venous duplex scanning and treatment procedures that are more simplified by minimally invasive, endovenous techniques. One such endovascular procedure called radiofrequency ablation (RFA) has been accepted in recent years as a favorable alternative to conventional surgery.

The successful treatment of lower extremity chronic venous insufficiency includes the elimination of all sources of venous reflux. The most common sources of reflux are superficial veins, which may or may not include the deep or perforator venous systems [2-4]. The primary objective in the treatment of superficial and perforator venous reflux disease is elimination of the refluxing veins from the venous circulation. The traditional surgical approach for saphenous vein reflux has been saphenous vein ligation at the saphenofemoral junction with ligation of all tributary vessels, with or without removal of the refluxing saphenous vein. Sclerotherapy of the incompetent saphenous vein has been found to be short lived and is not a recommended technique [5]. Reducing venous hypertension in this manner was not usually enough to eliminate truncal varicosities, and frequently required stab phlebectomy at the same time or later by sclerotherapy. Eliminating perforator vein reflux required open (Linton procedure) or endoscopic interruption of

* Vein Care Pavilion of the South, 447 North Belair Road, Suite 103, Evans, GA 30809.

E-mail address: vascularman40@yahoo.com 
abnormal venous flow (subfascial endoscopic perforator vein surgery or SEPS), and sclerotherapy with or without ultrasound guidance. With worldwide clinical experience increasing, endovenous RFA has become the most common alternative approach to superficial and perforator venous reflux disease [5].

\section{Radiofrequency ablation mechanism of action}

Endovenous RFA, also referred to as the VNUS Closure procedure (VNUS Medical Technologies Inc., San Jose, California) is a catheter-based endovascular intervention. The direction of RF energy into tissue to cause its destruction is safer and more controllable than other mechanisms used for this purpose. With RF energy delivered in continuous or sinusoidalwave mode, there is no stimulation of neuromuscular cells when a high frequency (between 200 and $3000 \mathrm{kHz}$ ) is used. The mechanism by which RF current heats tissue is resistive (or ohmic) heating of a narrow rim of tissue that is in direct contact with the electrode. Deeper tissue planes are then slowly heated by conduction from the small region of volume heating. Heat is dissipated from the region by further heat conduction into normothermic tissue. By regulating the degree of heating, subtle gradations of either controlled collagen contraction or total thermocoagulation of the vein wall can be achieved. The heat produced during RFA is caused by the resistance of the tissue in the veins wall allowing passage of the current. Therefore, during this resistive heating, the heat is generated in the vein wall and not in the catheter tip. A thermocouple on one of the electrodes applied to the vein wall measures the temperature, which can be kept constant by negative feedback. Clinically, the device produces precise tissue destruction with endothelial denudation, denaturing of the media and intramural collagen, with subsequent fibrotic seal of the vein lumen with minimal formation of thrombus and coagulum. Bipolar electrodes are used to heat the vein wall. The net effect is venous spasm and collagen shrinkage that produce maximal physical contraction [6]. The selective insulation of the electrodes results in a preferential delivery of the radiofrequency energy to the vein wall and minimal heating of the blood within the vessel, which eliminates vessel perforation.

The thermal effect on the vein wall is directly related to the treatment temperature and the treatment time, the latter being a function of catheter pullback speed. With a treatment temperature of $85^{\circ}$ to $90^{\circ} \mathrm{C}$ at a pullback speed of 3 to $4 \mathrm{~cm} / \mathrm{min}$, the thermal effect induced sufficient collagen contraction to occlude the lumen, while limiting heat penetration to perivenous tissue. In an in vitro model measuring changes in adventitial temperature along the vein, the highest temperature measured was $64^{\circ} \mathrm{C}$, and after a $2 \mathrm{~mm}$ tumescent fluid layer, this decreased to $51^{\circ} \mathrm{C}$. This emphasizes one of the benefits of tumescent fluid infiltration during the clinical procedure [7]. 
The other endovenous catheter-based thermoablation technique employs a laser system. Several companies now make laser systems that use variable wavelengths $(810 \mathrm{~nm}-1320 \mathrm{~nm})$. With laser, heat is generated by the action of the laser on the chromophore (substance that absorbs the laser light). It is thought that the mechanism of damage to the vein wall is the generation of a steam bubble around the laser tip from the blood still within the vein. This steam then transmits its heat to the vein wall via the high temperature gradient. The major chromophore at shorter wavelengths is hemoglobin, and longer wavelengths may have more of an action on water and collagen. The laser is fired and the vein wall is heated, probably via the steam bubble that is produced (boiling blood). There is no feedback, so control is by the amount of energy put into the fiber, the number and length of the pulses per centimeter if pulsed treatment is used, or the rate of pullback of the fiber if continuous laser energy is used. Thus there have been concerns that the variability of the amount of blood within the vein itself, the variability of the thickness of the vein wall, and the lack of any feedback information during treatment mean that it is difficult to know if one is under- or overtreating. Because this variability is more likely with laser and not RFA, laser treatment is, in the author's opinion, less likely to give consistently reproducible results and more likely to cause vessel perforation and patient discomfort and ecchymosis $[8,9]$.

\section{The Closure system and radiofrequency ablation technique}

Radiofrequency ablation is performed using the Closure catheter noted above. There are currently three types of catheters: (1) ClosurePLUS in both $6 \mathrm{Fr}$ and $8 \mathrm{Fr}$ for saphenous veins and accessory branches (Fig. 1); (2) radiofrequency system ClosureRFS and ClosurePLEX catheters for perforator veins (Fig. 2); and (3) the newly released ClosureFAST catheter for saphenous veins and accessory branches (Fig. 3). The ClosurePLUS catheter is designed for delivery of controlled RF to shrink vein wall collagen, and includes collapsible catheter electrodes around which the vein may shrink, and a central lumen to allow a guide wire or fluid delivery structured within a $5 \mathrm{Fr}(1.7 \mathrm{~mm})$ catheter. This design permits treatment of veins as small as $2 \mathrm{~mm}$ and as large as $24 \mathrm{~mm}$. The thermocouple on the electrode measures temperature and provides feedback to the RF generator. The control unit displays power, impedance, temperature, and elapsed time so that precise temperature control is obtained. The RF generator delivers the minimum power necessary to maintain the desired electrode temperature (Fig. 4).

Detailed procedure techniques have been described elsewhere [10,11]. The patient may be given oral or intravenous sedation and placed in a supine position on an adjustable table. The course of the greater saphenous vein (GSV) from the saphenofemoral junction to the knee, the lesser saphenous vein (LSV) from its junction with the gastrocnemic, popliteal, or other deep 

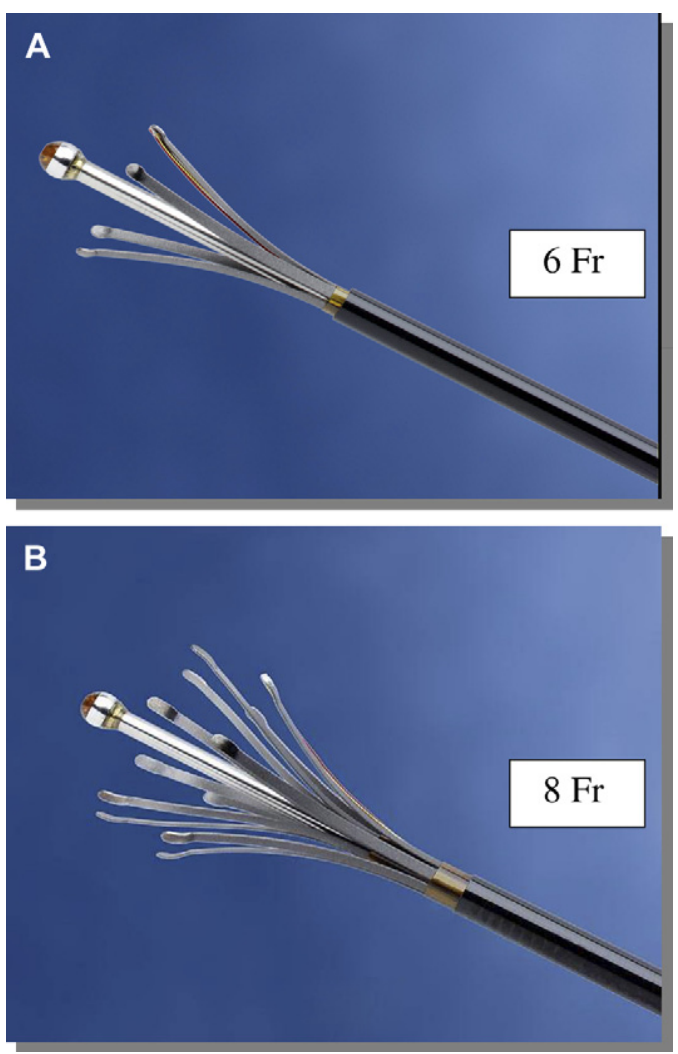

Fig. 1. VNUS ClosurePLUS $6 \mathrm{Fr}$ catheter $(A)$ and $8 \mathrm{Fr}$ catheter $(B)$. (Courtesy of VNUS Medical Technologies, San Jose, California; with permission.)

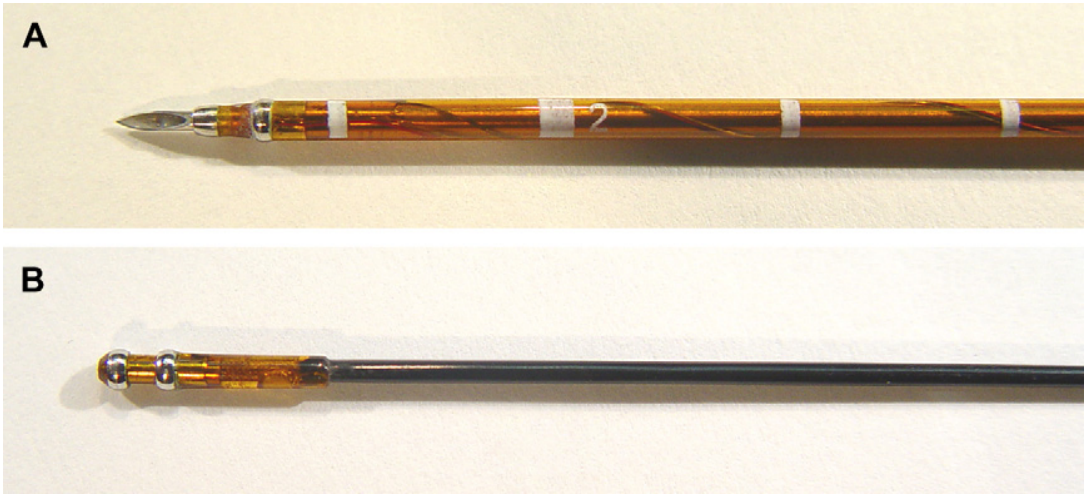

Fig. 2. VNUS ClosureRFS catheter $(A)$ and VNUS ClosurePLEX catheter $(B)$. (Courtesy of VNUS Medical Technologies, San Jose, California; with permission.) 


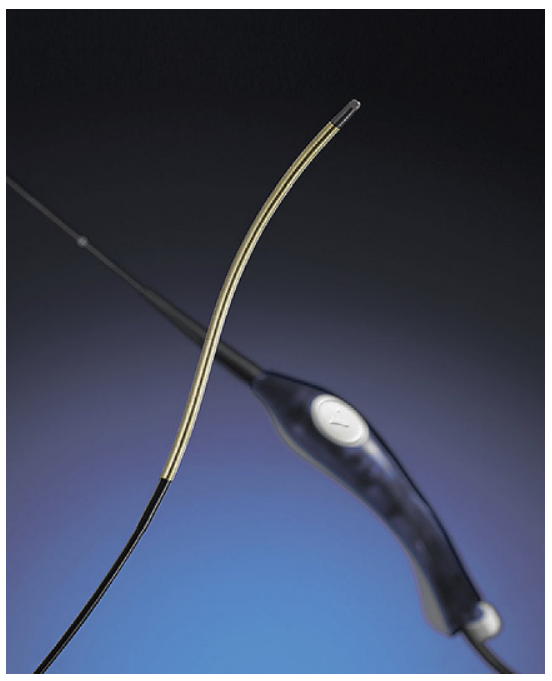

Fig. 3. VNUS ClosureFAST catheter. (Courtesy of VNUS Medical Technologies, San Jose, California; with permission.).

vein to the mid calf, and accessory branch (ie, anterior lateral branch) from its origin to the knee is mapped 6 to $8 \mathrm{~cm}$ apart using duplex ultrasound (Logic e, GE Medical Systems Ultrasound and Primary Care Diagnostics LLC, Milwaukee, Wisconsin) and marked with indelible ink. A patch of nitropaste may be applied to the planned access site before sterile surgical preparation to help dilate the vein and prevent venospasm (which can make percutaneous access difficult). Other venous dilation techniques may be applied as well (heating pad, tourniquet, reverse Trendelenberg position, and the like). After sterile preparation, the access sites and those sites marked previously are infiltrated with $1 \%$ buffered xylocaine without epinephrine. The vein is accessed using ultrasound guidance in transverse or longitudinal view with a 21 gauge needle that can accommodate a .018" wire. The smaller needle and wire are much more forgiving in causing
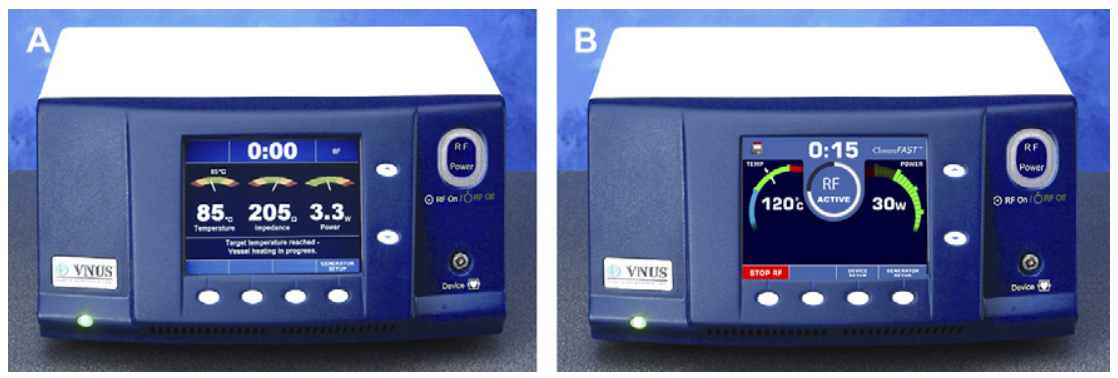

Fig. 4. Radiofrequency generator (RFG) PLUS screen with ClosurePLUS $(A)$ and ClosureFAST $(B)$. (Courtesy of VNUS Medical Technologies, San Jose, California; with permission.) 
venospasm than an 18 gauge needle and .035 " guide wire. Using the Seldenger technique, the introducer sheath ( $6 \mathrm{Fr}$ or $8 \mathrm{Fr}$ ) is advanced into the vein. A 6 or $8 \mathrm{Fr}$ catheter is advanced up to the appropriate end point. For the GSV, the catheter is delivered $1 \mathrm{~cm}$ below the ostium of the superficial epigastric vein. This position is confirmed by ultrasound (Fig. 5). The patient is placed into significant Trendelenberg position, and approximately 200 to $400 \mathrm{~mL}$ of tumescent fluid (normal saline, $1 \%$ xylocaine with epinephrine, bicarbonate) is injected directly, under ultrasound guidance, into the saphenous compartment from the insertion site up to $3 \mathrm{~cm}$ below the saphenofemoral junction. The tumescent solution provides local anesthetic, protects the surrounding tissues from heat-related damage, and compresses the vein around the catheter electrodes, ensuring complete vein wall treatment. The final position of the tip of the catheter is confirmed by ultrasound and the final injection of tumescent fluid is injected into the tissue surrounding the proximal $3 \mathrm{~cm}$ of the GSV where visualization will now be more obscured. To optimize exsanguination of the vein a circumferentially placed Esmark wrap can be applied. External compression to ensure contact between the vein wall and the electrodes and to maintain the temperature of the tip of the catheter at $85^{\circ}$ to $90^{\circ} \mathrm{C}$ can be achieved manually with the ultrasound probe. The withdrawal rate varies with desired treatment temperature. At conclusion of the procedure, the patency of the common femoral artery and common femoral vein, (popliteal, tibial, and gastrocnemic veins with the LSV), successful contraction of the GSV with a residual diameter of

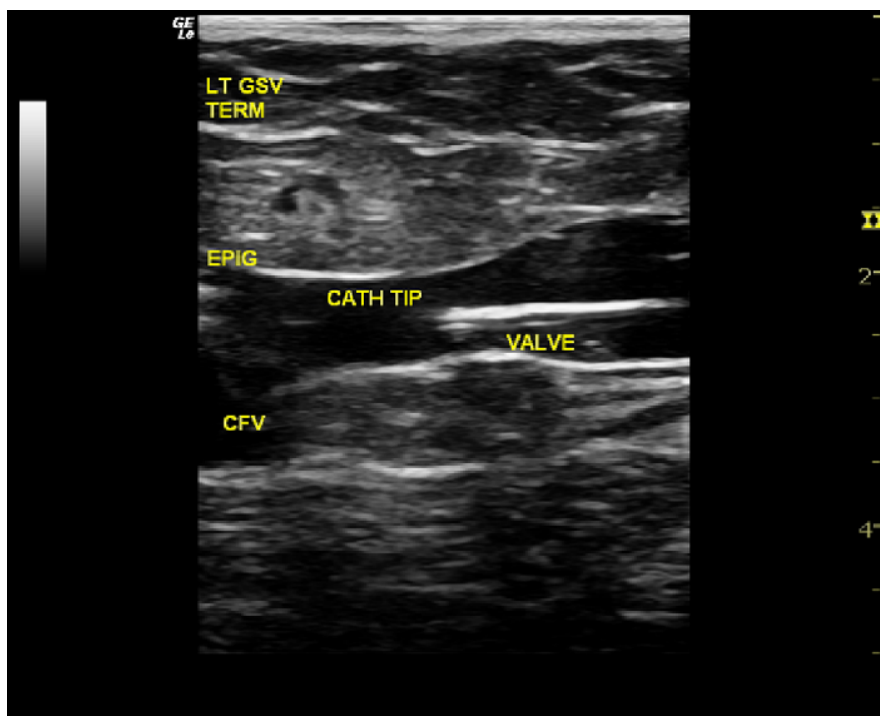

Fig. 5. Tip of the ClosureFAST catheter (CATH TIP) at the saphenofemoral junction $1 \mathrm{~cm}$ distal to the origin of the lateral superficial epigastric vein (EPIG). CFV, common femoral vein; GSV, greater saphenous vein. 
less than $2 \mathrm{~mm}$, and flow through the saphenofemoral junction (SFJ) into the lateral epigastric vein are confirmed by duplex examination (Fig. 6). Patients are then placed in elastic wrap bandages, and asked to wear graduated compression stockings with at least $30 \mathrm{~mm} / \mathrm{Hg}$ of compression and to walk at least 30 minutes per day. They are told to resume normal activity immediately and encouraged to resume aerobic exercise, but to avoid weight lifting for the next 2 or 3 days. A duplex ultrasound scan should be performed within 72 hours postoperatively to rule out any evidence of deep vein thrombosis (DVT). The procedure is commonly performed on an outpatient basis in an office setting, minor procedure room, ambulatory surgical center, or a traditional operating room.

The technique required for perforator vein RFA requires more detailed mapping than for superficial veins. Because the perforator veins are typically not linear in orientation like the superficial veins, and are more curvilinear and angulated throughout their course, adequate visualization with duplex ultrasound to allow for easy access can be challenging. Once an adequate approach angle has been identified and the access site marked, similar preparations are made as noted above. A rigid radiofrequency stylet (Closure RFS) catheter or more flexible catheter (ClosurePLEX) may be used. The perforator vein is accessed directly with the ClosureRFS catheter using duplex ultrasound. A 21 gauge access needle and .018" guide wire are used to access the perforator vein using the Seldinger technique when the ClosurePLEX is used. Either catheter is advanced to the level of the muscular fascia.

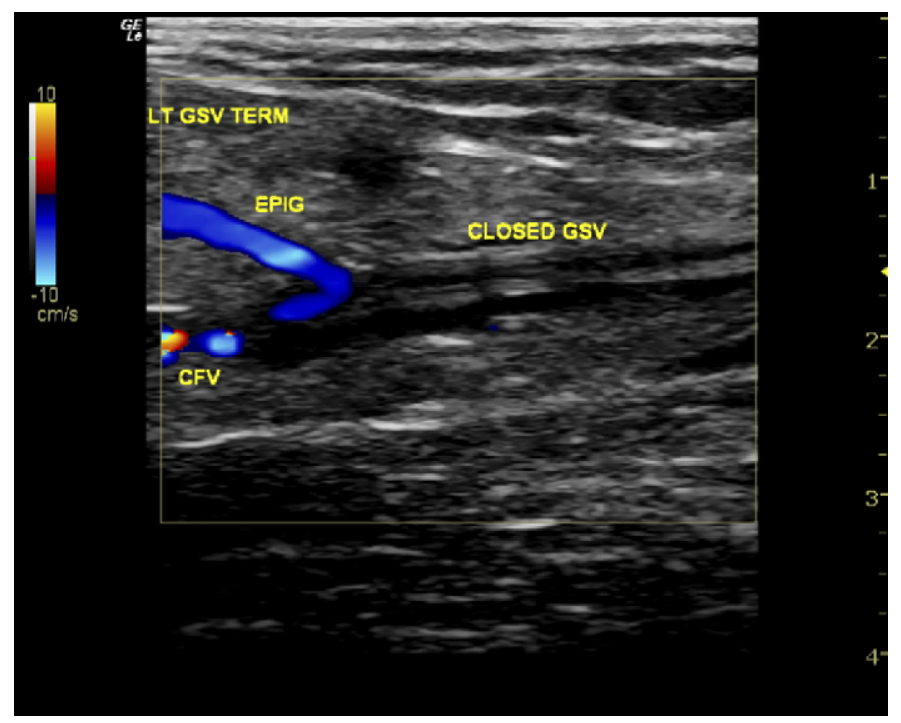

Fig. 6. Flow from common femoral vein (CFV) through terminal greater saphenous vein (LT GSV TERM) and the lateral superficial epigastric vein (EPIG), greater saphenous vein (GSV) diameter $<2 \mathrm{~mm}$, and no flow demonstrated through the GSV (CLOSED GSV). 
When the stylet is removed, the catheter tip of the ClosureRFS catheter will be in the proper position (5-10 $\mathrm{mm}$ from the fascia), whereas the ClosurePLEX should be pulled back 5 to $10 \mathrm{~mm}$. The tip of the catheter is held in this position, tumescent fluid infiltration introduced above and below the catheter tip, and the patient placed in Trendelenberg position. The treatment temperature can be $85^{\circ}$ to $90^{\circ} \mathrm{C}$ for 5 minutes, pulling back 1 to $10 \mathrm{~mm}$ during the last 1 minute. During the first 4 minutes the catheter tip is rotated to the 12, 6, 3, and 9 o'clock positions, each for 1 minute of treatment. An alternative technique uses a 12 gauge angiocatheter to initially access the perforator vein with duplex ultrasound and act as a portal for the ClosureRFS or ClosurePLEX catheters. Once the treatment is completed, the deep veins and arteries are assessed for patency, the catheters removed, and pressure is applied. Patients have a stretch wrap applied to the leg with a graduated compression stocking of at least $30 \mathrm{~mm} / \mathrm{Hg}$, and are asked not to remove it for 3 to 4 days. Patients are expected to resume normal activity just as with the superficial veins, and a follow-up scan is done within 72 hours.

Although SEPS has recently been the most common way to treat incompetent perforator veins, there are several potential advantages of using ultrasound-guided endovenous RFA. The first is that the intervention is truly a minimally invasive procedure that can be performed in the office. Unlike SEPS, the approach is not limited by the perforating vein location. With RFA technique, the physician can access the incompetent perforator veins (IPVs)at various positions including the more proximal locations (Boyd's, Dodd's, Hunter's, lateral perforators, perimalleolar). The procedure also allows the flexibility of repeat treatment for persistent or newly developed IPVs. The use of RFA is generally reserved for patients who have advanced chronic venous insufficiency with clinical, etiologic, anatomic, and pathophysiologic (CEAP) classes 4 to 6 , although it can be performed as part of the primary treatment for varicose veins, and is useful for treating recurrent varicose veins.

\section{Radiofrequency ablation safety}

The VNUS Closure system received US Food and Drug Administration (FDA) approval in March 1999. To date, there have been over 100,000 RFA procedures performed worldwide, and its safety has been carefully reported [12-14]. Most impressively, a clinical registry was established in 1998 to monitor procedure safety and treatment outcomes. More than 30 centers worldwide contributed data, and RFA has the largest collection of published data in endovenous surgery, including registry data regarding endovenous therapy with 5 year follow-up, and both single center data and multicenter randomized trials comparing RFA with vein stripping.

Modifications in the RFA procedure were made as data were reported. Tumescent infiltration was initiated to reduce the risk of skin burns, which are rarely observed now. DVT is a potential risk for any surgical procedure. In the setting of RFA, DVT can originate from the treated superficial or 
perforator vein and extend into the deep venous system. Attention is drawn to careful tip positioning to ensure that treatment begins a short distance from the SFJ, and to preserve the physiological blood flow from the superficial epigastric tributary. Immediate and sufficient ambulation is encouraged in addition to the follow-up ultrasound scan within 72 hours. The DVT rates were reported to be $0 \%$ to $1 \%$ in most series that have been published. In one series [15], DVT was detected in 12 of 73 limbs (16.4\%), which is felt to be an aberrancy from the experiences of others $[16,17]$.

Paresthesia was reported to occur in $9 \%$ to $19 \%$ of limbs within 1 week after the procedure [12-14,18-27], and gradually resolved over time [25]. Limiting treatment to the above-knee segment decreases the risk of paresthesia because $90 \%$ of the time the saphenous nerve travels within the saphenous compartment from the groin to the knee, and then travels superficial to this compartment, and thus is more susceptible to injury with heat in the below-knee location despite tumescent fluid infiltration. Similarly, with RFA treatment of the LSV, the peroneal nerve and superficial branches of the sural nerve emerge around mid-calf level and should be avoided [7].

\section{Treatment efficacy and outcomes}

Pichot and colleagues [28] have described the morphological and hemodynamic outcomes following RFA using detailed duplex ultrasound. There is a sonographic progression observed in the pathologic sequelae of the treated vein. Veins closed completely were initially hypoechogenic compared with the surrounding tissue, and progressed into a hyperechogenic state and finally became isoechogenic, indicating a healing process. Approximately $60 \%$ of veins were hypoechogenic and $40 \%$ were hyperechogenic at 1 week. At 6 months, they became either hyperechogenic or isoechogenic. Weiss [19] demonstrated the sonographic disappearance of the saphenous vein in $90 \%$ of limbs after 2 years.

Anatomical failure is defined as partial or nonocclusion of a treated vein resulting from incomplete treatment or subsequent recanalization. It has been demonstrated that even with anatomical failure, clinical improvement was often demonstrated in patients [25]. The strong possibility of later recurrence in these patients was also evident.

Morphologic differences in the SFJ following RFA have been studied. Four types of SFJ morphologies were identified [25,28,29]. The three most common include: J-1, defined as complete SFJ obliteration with no SFJ flow; J-2 as patent SFJ tributaries draining toward the femoral vein with or without a short saphenous stump; and J-3 as terminal GSV competence with normal antegrade flow coming from both tributaries and the saphenous vein above a limited GSV obliteration. Two years after RFA, the most common findings were either complete SFJ obliteration or a $5 \mathrm{~cm}$ or less patent terminal stump conducting antegrade tributary flow through the SFJ, accounting for approximately $90 \%$ of the limbs treated $[25,29]$. 
The clinical significance of a short patent SFJ stump was analyzed by Merchant and colleagues [25]. A total of 319 limbs (286 patients) in the clinical registry were followed up at 1 week, 6 months, 1 year, and 2 years, with 121 limbs having 2-year data. Symptom improvement and varicose vein absence demonstrated no statistically significant differences between patients who have complete SFJ obliteration and those who have a short patent SFJ stump at any follow-up time points. Although the distal trunk is occluded, SFJ competence is often restored, even with a short patent stump. A patent stump can serve a conduit and preserve the normal physiologic flow from one or more patent tributaries, such as those draining blood from abdominal and pudendal areas. Preservation of this physiological flow has been considered to be an advantage of endovenous procedures over traditional veins stripping because it causes less hemodynamic disturbance. More hemodynamic disturbance is thought to be one of the factors responsible for stimulating neovascularization following vein stripping.

RFA treatment results for large veins were studied by Merchant and colleagues [30]. In the 5-year registry data, there were 39 veins with diameter greater than $12 \mathrm{~mm}$ (maximum diameter $24 \mathrm{~mm}$ ). Vein occlusion rate was $97 \%$ within 1 week and $92.6 \%$ at 6 months and 1 year. The author has had similar experience (Fig. 7).

The efficacy of RFA treatment has been reported by several groups [19-27]. The largest study with the longest follow-up is the clinical registry series. A 90\% reflux-free rate was reported at 2 years [15]. The latest available results with 5-year follow-up data demonstrated RFA treatment efficacy of $84 \%$ compared with $89 \%$ at 1 year, showing the durability of the treatment. It is important to note that in the registry the patients who have longer follow-ups are those treated at the very early stages of RFA. Because the RFA procedure technique has improved compared with earlier treatments, treatment efficacy has improved.

Patient symptom relief has been reported by several studies. Approximately $85 \%, 79 \%$, and $39 \%$ of limbs had pain, fatigue, or edema, respectively, before the procedure; these decreased to $29 \%, 7 \%$, and $8 \%$ within 1 week after the procedure. A further decrease of the percentage of symptomatic limbs was observed at 6 months, and the symptom improvement remained stable over 5 years. Pullback speed less than $3 \mathrm{~cm} / \mathrm{min}$ and body mass index (BMI) greater than $25 \mathrm{~m} / \mathrm{kg}^{2}$ were identified as risk factors for anatomical failure. These results have established the long-term treatment outcomes of RFA in eliminating saphenous reflux and alleviating patient symptoms.

\section{Endovenous radiofrequency ablation versus vein stripping}

The premise behind both RFA and traditional vein stripping is to remove the incompetent vein from the venous circulation to reduce the venous hypertension, with subsequent resolution of symptoms without significant morbidity. The advantages of RFA over vein stripping are demonstrated 

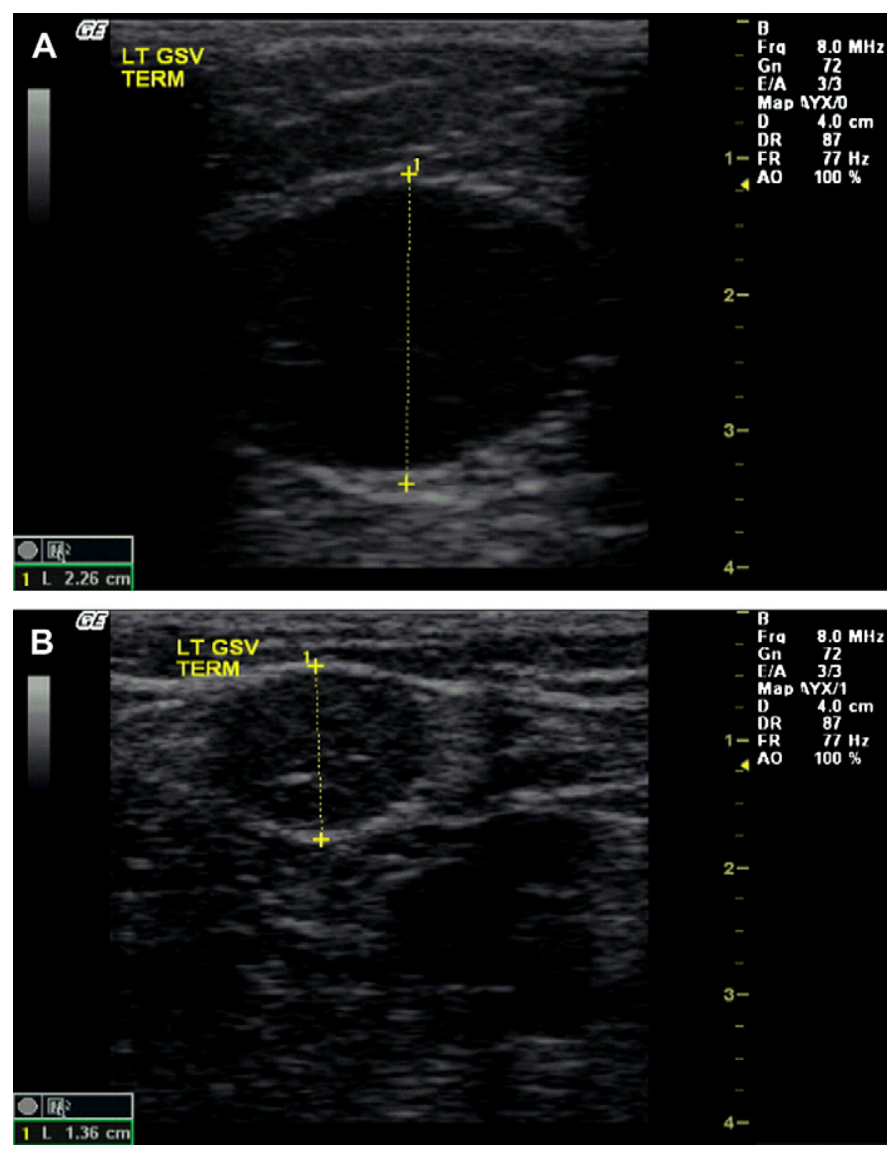

Fig. 7. (A) Before VNUS Closure was performed on large greater saphenous vein (GSV), it measured $22.6 \mathrm{~mm}$. (B) Three months after VNUS closure, the terminal GSV (LT GSV TERM) had no flow and measured $13.6 \mathrm{~mm}$.

in three randomized comparative trials. Rautio and colleagues [20] reported significantly less postoperative pain, quantitated with a visual analog scale, in the RFA group than in the stripping group at rest, on standing, and on walking, with the most distinct differences between the 5 th to the 14 th postoperative days. The analgesics needed in the RFA patients were statistically less for the stripping group. Sick leaves were also significantly shorter in the RFA group, and physical function was restored faster in the RFA patients, measured with the RAND-36 generic health-related quality of life (QOL) questionnaire. All the operations were successful and the complications were similar between the two groups in this study. These patients were followed-up at 3 years [31]. Venous clinical severity scores (VCSS) were similar for both groups. None of the RFA-occluded GSVs were recanalized. Varicose vein recurrence rate was documented in 5 of 15 limbs (33\%) in the RFA 
group and 3 of 13 limbs (23\%) in the stripping group, and the difference was not statistically significant. Similarly, Stötter and colleagues [21], in their randomized trial in Germany, reported a significantly lower pain score in the RFA group versus the stripping group within 6 weeks following the procedure.

RFO and vein stripping were further compared on bilaterally recurrent patients [32]. Sixteen patients who had bilateral recurrent GSV reflux were randomly treated with RFA on one leg and with vein stripping on the other leg. The RFA was faster (25 minutes versus 40 minutes), associated with significantly less postoperative pain and bruising, and had higher patient preference. It was concluded that RFA should be the treatment of choice for recurrent saphenous vein reflux.

The VNUS company sponsored a multicenter prospective randomized trial called EndoVenous Obliteration versus Ligation and Vein Stripping (EVOLVES). The early follow-up (within 4 months) focused on the comparison of the procedure related complications, patient recuperation, and quality of life outcomes between the two treatment procedures, and the procedure impact on the hemodynamic and clinical outcomes was demonstrated through 1 and 2 year follow-up evaluations [22,23]. Patients who had symptomatic varicose veins and GSV incompetence were randomized into either the RFA or the vein stripping group. RFA was performed on 44 limbs and stripping on 36 limbs. There were no differences between the groups in patient demographics, VCSS, or CEAP clinical class distribution before the treatment $[25,33]$. The most significant differences seen between the two groups were on patient recovery. The mean time to return to normal activity was 1.15 days for the RFA patients compared with 3.89 days $(P=.02)$ for vein stripping. There were $80.5 \%$ of RFA patients who returned to routine activities within 1 day, versus $46.9 \%$ of patients with stripping $(P<.01)$. Mean values for the time to return to work were 4.7 days in the RFA group and 12.4 days in the stripping group $(P=.05)$. Of note, there were significantly fewer complications and adverse advents observed in the RFA group through 3 weeks postoperatively, mostly because of higher rates of tenderness, ecchymosis, and hematoma following vein stripping. The postoperative VCSS were significantly different at 72 hours and 1 week, but the difference disappeared at later follow-up. The QOL surveys (CIVIQ2 questionnaire) showed clearly superior patient QOL in the RFA patients; the most significant differences were seen in global, pain, and physical scores. The pain score improvement form pretreatment baseline was significantly better in RFO than in the stripping patients at all the follow-up time points out to 2 years. The differences observed in global scores were statistically significant at 72 hours and 1 week, and surprisingly also at 1 and 2 years. Clinical and hemodynamic outcomes were similar for both groups at 2 years. There were no differences in patient symptoms and signs, and no differences on varicose vein recurrence rates. Duplex ultrasound examination revealed $91.7 \%$ and $89.7 \%$ of limbs free of reflux in the RFA and vein stripping 
groups, respectively. At 2 years, neovascularization was reported in 1 RFA limb $(2.8 \%)$ and 4 vein stripping limbs $(13.8 \%)(P>.05)[25,33]$.

The lower incidence of neovascularization with RFA was also reported by Pichot and colleagues [29]. Sixty three limbs were studied with ultrasound scan protocol and found to have no evidence of neovascularization at 2 years after the treatment. Two advantages of RFA that are thought to account for the low incidence of neovascularization are: (1) no incision and surgical dissection of the groin, and (2) minimal hemodynamic disturbance because of preservation of physiologic abdominal wall drainage. Recurrence can refer to either clinical recurrence (ie, varicose vein recurrence) or hemodynamic recurrence (ie, SFJ reflux). Varicose vein recurrence rates after vein stripping have been reported between $20 \%$ and $50 \%$ at 2 to 5 years [34-41] and up to $70 \%$ by 10 years [42]. The varicose vein recurrence rate is more subjective; the recurrence of SFJ reflux is a more objective measure and provides important hemodynamic information that permits detection and prediction of clinical recurrence. It was reported that $87 \%$ of limbs were free from reflux at 2 years and $71 \%$ were reflux free at 5 years after vein stripping [40]. Vein ligation without stripping achieved only $58 \%$ freedom from reflux at 2 years, and only $29 \%$ freedom from reflux at 5 years. Fischer and colleagues [43] studied 125 limbs after ligation of the true saphenofemoral junction and its related tributaries, and found that $60 \%$ of limbs had SFJ reflux at mean follow-up of 3.4 years.

\section{New technology with endovenous ablation}

The VNUS ClosureFAST catheter is the next generation of closure catheter that was developed to improve the procedure speed and the ease of use compared with the current Closure catheter. The catheter consists of a flexible catheter shaft and a $7 \mathrm{~cm}$ long distal heating element. The heating element has a fixed diameter and is covered with a glide cover to prevent sticking after heating. Good contact between the catheter and the vein wall is established by tumescent infiltration, Trendelenberg position, and external compression, and is monitored by an external thermocouple on the catheter and the computer software in the RF generator. The temperature of the heating element is monitored and controlled by a temperature sensor that regulates the amount of energy delivered during the treatment. Unlike the current Closure and endovenous laser devices that involve continuous pullback, the ClosureFAST catheter uses segmental heating approach. Once the catheter is positioned and the vein appropriately compressed, the heating element is activated with RF energy for the duration of the 20-second heating cycle. The $7 \mathrm{~cm}$ element heats to $120^{\circ} \mathrm{C}$, allowing for a $0.5 \mathrm{~cm}$ overlap between each treated segment to ensure complete treatment along the length. The new catheter does not require a saline drip, which is different from the previous VNUS catheters, and it appears to completely eliminate the high 
impedance issues caused by coagulum buildup with the previous catheter. After completion of the 20 -second heating cycle, the catheter is positioned to the next segment, guided by the shaft markers, and treatment starts again. Accurate pullback is facilitated by positioning the sheath to align with one of the $6.5 \mathrm{~cm}$ markers on the catheter shaft. Two heating cycles are applied to the first segment near the SFJ to ensure sufficient treatment of this important segment (Fig. 8) [44].

The clinical experience with the ClosureFAST catheter comes from Europe. More than 200 limbs were treated, with the longest follow-up being 6 months. All treated veins remained closed at the latest follow-up, confirmed by ultrasound examinations. There were no DVTs, skin burns, or other serious adverse events. The average treatment time was 2.2 minutes, with an average treatment length of $37 \mathrm{~cm}$. The average time from catheter insertion to catheter removal was approximately 16 minutes [44].

The new procedure is well-tolerated by patients, and there was no noticeable difference in patient reaction to this procedure compared with the current Closure procedure. Postprocedural recovery continued to be benign;
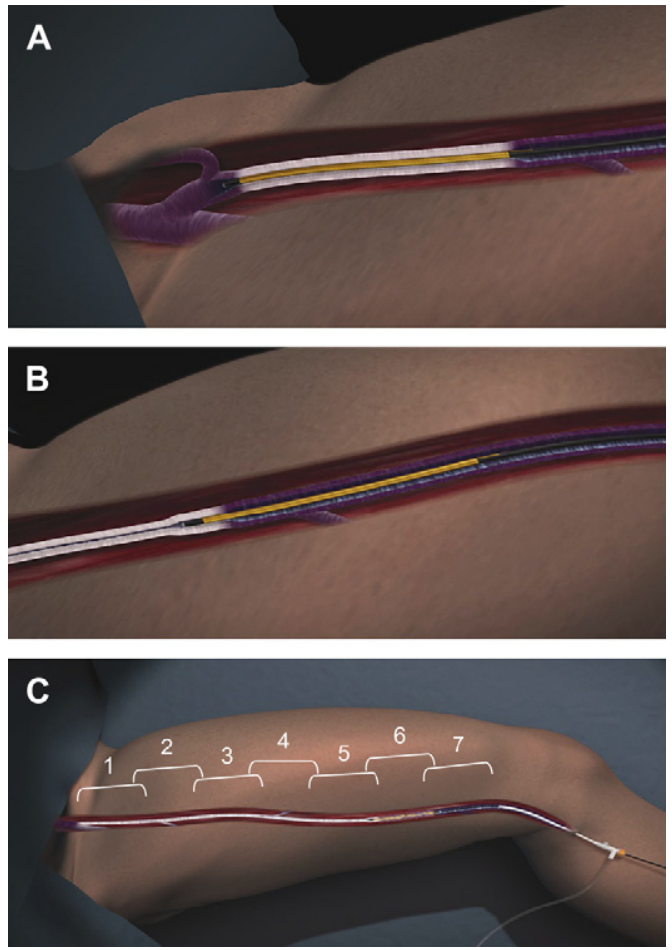

Fig. 8. (A) ClosureFAST catheter stationary during treatment at sapheno-femoral junction. $(B)$ Slight overlap between treatments. $(C)$ Vein treated stepwise along the length. (Courtesy of VNUS Medical Technologies, San Jose, California; with permission.) 
$79 \%$ of limbs experienced no pain and $87 \%$ experienced no tenderness. The experience of Lumsden and Peden [44] reported results that concurred with those of the European study. This is an important distinction between RFA and endovenous laser (EVL). After EVL, $67 \%$ to $100 \%$ of patients reported to have some degree of pain, with more than $50 \%$ of patients requiring analgesics for pain management, and a $10 \%$ to $33 \%$ rate of thrombophlebitis. The pain, bruising, and thrombophlebitis are thought to be the result of vein wall perforation and the thrombotic mechanism associated with EVL [45-47].

With the new device, a $40 \mathrm{~cm}$-long vein segment can be treated stepwise in less than 3 minutes, which is equal to or faster than EVL protocols. The rate of immediate vein occlusion at the saphenofemoral junction is $100 \%$, a value not reached previously by most users of laser and RFA. The limited number of side effects is clearly the same as with previous RFA procedures, but not nearly as high as with laser treatment.

\section{Comparison of endoluminal techniques}

Endovenous radiofrequency ablation is a new endoluminal treatment for those patients who have superficial and perforator venous reflux disease. These endoluminal techniques are challenging the traditional methods of open surgery that have been used for decades in treating primary varicose vein disease and venous stasis ulcer disease. The other endoluminal technique, EVL, can only be used for superficial venous reflux disease and cannot be used safely for perforator venous reflux disease. EVL has not been studied as thoroughly as RFA in comparing the endoluminal technique with open surgery [48,49]. The longest follow-up with clinical results after EVL has been 2 years, whereas detailed results are available at 5 -year follow-up for RFA. There is no randomized controlled trial comparing RFA with EVL or sclerotherapy with endoluminal thermal obliteration; however, a randomized controlled trial comparing RFA to EVL demonstrated better primary obliteration and less postoperative pain and bruising with RFA [50]. In terms of thromboembolic events after endoluminal treatment, RFA and EVL presented an approximate equal rate of $0.5 \%$. Vein closure after RFA was $87.2 \%$ in a multicenter study, and varicose vein recurrence was $27.4 \%$ at 5 year follow-up; vein obliteration after EVL was reported by various groups to be $76 \%$ to $96.8 \%$ at 1 - to 2 -year follow-up [50].

One of the advantages usually credited to EVL treatment is that the duration of the procedure is shorter than that for RFA because of the faster pullback speed. The new VNUS ClosureFAST catheter has eliminated this advantage. A cost comparison of the two techniques indicates that although the EVL fiber is cheaper than the RF catheter, the cost of the generator is less for the RF procedure [51]. 


\section{Summary}

Endovenous radiofrequency obliteration has become the favored alternative choice in the treatment of superficial and perforator venous reflux disease. RFA not only outperforms traditional vein stripping and perforator interruption with regard to morbidity and outcome, it reduces the incidence of neovascularization that is frequently blamed for the higher recurrence rates with vein stripping. RFA has been shown to have fewer postprocedural complications than laser treatment. Some of the previous shortcomings of the VNUS ClosurePLUS catheter, including high impedance failure, coagulum buildup, and slower pullback speed, have been eliminated with the new ClosureFAST catheter. This new catheter system is an important advancement that combines the faster withdrawal speed of laser treatment with the fewer side effects and high ablation rate commonly seen with RFA.

\section{Acknowledgments}

The author would like to recognize the help of Mr. Ralph Roper, RVT for his assistance with the preparation of the ultrasound images, as well as all the staff of the Vein Care Pavilion of the South and the Vein Care Pavilion of America.

\section{References}

[1] Callam MJ. Epidemiology of varicose veins. Br J Surg 1994;81:167-73.

[2] Ruckley CV, Evans CJ, Allan PL, et al. Chronic venous insufficiency: clinical and duplex correlations. The Edinburgh Vein Study of Venous Disorders in the General Population. J Vasc Surg 2002;36:520-5.

[3] Labropoulos N, Delis K, Nicolaides AN, et al. The role of the distribution and anatomic extent of reflux in the development of signs and symptoms in chronic venous insufficiency. J Vasc Surg 1996;23(3):504-10.

[4] Weiss RA. RF-mediated endovenous occlusion. In: Weiss RA, Feied CF, Weiss MA, editors. Vein diagnosis and treatment: a comprehensive approach. New York: McGraw-Hill Medical Publishing Division; 2001. p. 211-21.

[5] Bergan JJ. Endovenous sphenous vein obliteration. In: Whittmore AD, Bandyk DF, editors. Advances in vascular surgery, vol. 9. Chicago: Mosby-Year Book; 2001. p. 123-32.

[6] Nicolaides AN, Griffin MB, Lennox AF, et-al. Endovenous vein closure. In: Greenhalgh RM Becquemin JP Raithel D, et-al, editors. Vascular and endovascular surgical techniques. 4th edition. Philadelphia: WB Saunders; 2001. p. 507-10.

[7] Zikorus AW, Mirizzi MS. Evaluation of setpoint temperature and pullback speed on vein adventitial temperature during endovenous radiofrequency energy delivery in an in-vitro model. Vasc Endovascular Surg 2004;38:167-74.

[8] Min RJ, Zimmet SE, Isaacs MN, et al. Endovenous laser treatment of the incompetent greater saphenous vein. J Vasc Interv Radiol 2001;12:1167-71.

[9] Proebstle TM. Comments on "Comparison of endovenous radiofrequency versus 810 $\mathrm{nm}$ diode laser occlusion of large veins in animal model." Dermatol Surg 2002;28: 596-600. 
[10] Weiss RA. RF-mediated endovenous occlusion. In: Weiss RA, Feied CF, Weiss MA, editors. Vein diagnosis and treatment: a comprehensive approach. 1st edition. St. Louis (MO): Quality Medical Publishing; 1999. p. 217-24.

[11] Weiss RA. Radiofrequency endovenous occlusion (Closure Technique). In: Frenek HS, editor. Fundamentals of phlebology: venous disease for clinicians. 2nd edition. London: Royal Society of Medicine Press Ltd.; 2002. p. 101-4.

[12] Manfrini S, Gasbarro V, Danielsson G, et al. Endovenous management of saphenous vein reflux. J Vasc Surg 2000;32:330-42.

[13] Chandler JG, Pichot O, Sessa C, et al. Treatment of primary venous insufficiency by endovenous saphenous vein obliteration. Vasc Surg 2000;34:201-14.

[14] Dauplaise TL, Weiss RA. Duplex-guided endovascular occlusion of refluxing saphenous veins. Journal of Vascular Technology 2001;25:79-82.

[15] Sybrandy JEM, Wittens CHA. Initial experiences in endovenous treatment of saphenous vein reflux. J Vasc Surg 2002;36:1207-12.

[16] Hingorani AP, Ascher E, Markevich N, et al. Deep venous thrombosis after radiofrequency ablation of greater saphenous vein: a word of caution. J Vasc Surg 2004;40(3):500-4.

[17] Subramonia S, Lees T. Regarding "Deep vein thrombosis after radiofrequency ablation of greater saphenous vein: a word of caution." J Vasc Surg 2005;41(5):915-6.

[18] Chandler JG, Pichot O, Sessa C, et al. Defining the role of extended saphenofemoral junction ligation: a prospective comparative study. J Vasc Surg 2000;32:941-53.

[19] Weiss RA, Weiss MA. Controlled radiofrequency endovenous occlusion using a unique radiofrequency catheter under duplex guidance to eliminate saphenous varicose vein reflux: a 2-year follow-up. Dermatol Surg 2002;28:38-42.

[20] Rautio T, Ohinmaa A, Perälä J, et al. Endovenous obliteration versus conventional stripping operation in the treatment of primary varicose veins: a randomized controlled trial with comparison of costs. J Vasc Surg 2002;35:958-65.

[21] Stötter L, Schaaf I, Bockelbrink A, et al. [Radiofrequency obliteration, invagination, or cryostripping: which is the best tolerated by the patients?]. Phlebologie 2005;34:19-24 [in German].

[22] Lurie F, Creton D, Eklof B, et al. Prospective Randomized Study of Endovenous Radiofrequency Obliteration (Closure) versus Ligation and Stripping in a selected patient population (EVOLVES study). J Vasc Surg 2003;38:207-14.

[23] Lurie F, Creton D, Eklof B, et al. Prospective Randomised Study of Endovenous Radiofrequency Obliteration (Closure) Versus Ligation and Vein Stripping (EVOLVeS): two-year follow-up. Eur J Vasc Endovasc Surg 2005;29(1):67-73.

[24] Goldman MP, Amiry S. Closure of the greater saphenous vein with endoluminal radiofrequency thermal heating of the vein wall in combination with ambulatory phlebectomy: 50 patients with more than 6-month follow-up. Dermatol Surg 2002;28:29-31.

[25] Merchant RF, DePalma RG, Kabnick LS. Endovascular obliteration of saphenous reflux: a multicenter study. J Vasc Surg 2002;35(6):1190-6.

[26] Rautio TT, Perälä JM, Wiik HT, et al. Endovenous obliteration with radiofrequencyresistive heating for greater saphenous vein insufficiency: a feasibility study. J Vasc Interv Radiol 2002;13:569-75.

[27] Gale SS, Dosick SM, Seiwert AJ, et al. Regarding "Deep venous thrombosis after radiofrequency ablation of greater saphenous vein." J Vasc Surg 2005;41(2):374.

[28] Pichot O, Sessa C, Chandler JG, et al. Role of duplex imaging in endovenous obliteration for primary venous insufficiency. J Endovasc Ther 2000;7:451-9.

[29] Pichot O, Kabnick LS, Creton D, et al. Duplex ultrasound scan findings two years after great saphenous vein radiofrequency endovenous obliteration. J Vasc Surg 2004;39:189-95.

[30] Merchant RF, Pichot O, Mayers KA. Four-year follow-up on endovascular radiofrequency obliteration of great saphenous reflux. Dermatol Surg 2005;31:129-34.

[31] Perala J, Rautio T, Biancari F, et al. Radiofrequency endovenous obliteration versus stripping of the long saphenous vein in the management of primary varicose veins: 3-year outcome of a randomized study. Ann Vasc Surg 2005;19:1-4. 
[32] Hinchliffe RJ, Ubhi J, Beech A, et al. A prospective randomised controlled trial of VNUS closure versus surgery for the treatment of recurrent long saphenous varicose veins. Eur J Vasc Endovasc Surg 2006;31(2):212-8.

[33] Kistner RL, Eklof B, Masuda EM. Diagnosis of chronic venous disease of the lower extremities: the "CEAP" classification. Mayo Clin Proc 1996;71:338-45.

[34] Dwerryhouse S, Davies B, Harradine K, et al. Stripping the long saphenous vein reduces the rate of reoperation for recurrent varicose veins: five-year results of a randomized trial. J Vasc Surg 1999;29:589-92.

[35] Rutgers PH, Kitslaar PJ. Randomized trial of stripping versus high ligation combined with sclerotherapy in the treatment of the incompetent greater saphenous vein. Am J Surg 1994; 168:311-5.

[36] Neglen P, Einarsson E, Eklof B. The functional long-term value of different types of treatment for saphenous vein incompetence. J Cardiovasc Surg (Torino) 1993;34:295-301.

[37] Munn SR, Morton JB, Macbeth WA, et al. To strip or not to strip the long saphenous vein? A varicose veins trial. Br J Surg 1981;68:426-8.

[38] Hammarsten J, Pedersen P, Cederlund CG, et al. Long saphenous vein saving surgery for varicose veins. A long-term follow-up. Eur J Vasc Surg 1990;4:361-4.

[39] Jones L, Braithwaite BD, Selwyn D, et al. Neovascularisation is the principal cause of varicose vein recurrence: results of a randomised trial of stripping the long saphenous vein. Eur J Vasc Endovasc Surg 1996;12:442-5.

[40] van Rij AM, Jiang P, Solomon C, et al. Recurrence after varicose vein surgery: a prospective long-term clinical study with duplex ultrasound scanning and air plethysmography. J Vasc Surg 2003;38:935-43.

[41] Kostas T, Ioannou CV, Touloupakis E, et al. Recurrent varicose veins after surgery: a new appraisal of a common and complex problem in vascular surgery. Eur J Vasc Endovasc Surg 2004;27:275-82.

[42] Campbell WB, Vijay Kumar A, Collin TW, et al. The outcome of varicose vein surgery at 10 years: clinical findings, symptoms and patient satisfaction. Randomised and economic analysis of conservative and therapeutic interventions for varicose veins study. Ann R Coll Surg Engl 2003;85:52-7.

[43] Fischer R, Linde N, Duff C, et al. Late recurrent saphenofemoral junction reflux after ligation and stripping of the greater saphenous vein. J Vasc Surg 2001;34(2):236-40.

[44] Lumsden AB, Peden EK. Clinical use of the new VNUS ClosureFAST radiofrequency catheter. Endovascular Today 2007;(Suppl):7-10.

[45] Proebstle TM, Lehr HA, Kargl A, et al. Endovenous treatment of the greater saphenous vein with a 940-nm diode laser: thrombotic occlusion after endoluminal thermal damage by lasergenerated steam bubbles. J Vasc Surg 2002;35:729-36.

[46] Proebstle TM, Krummenauer F, Gul D, et al. Nonocclusion and early reopening of the great saphenous vein after endovenous laser treatment is fluence dependent. Dermatol Surg 2004; 30:174-8.

[47] Kabnick LS. Outcome of different endovenous laser wavelengths for great saphenous vein ablation. J Vasc Surg 2006;43:88-93.

[48] de Medeiros CA, Luccas GC. Comparison of endovenous treatment with an 810-nm laser versus conventional stripping of the great saphenous vein in patients with primary varicose veins. Dermatol Surg 2005;31:1685-94.

[49] Vuylsteke M, Van den Bussche D, Audenaert EE, et al. Endovenous laser obliteration for the treatment of primary varicose veins. Phlebology 2006;21:80-7.

[50] Morrison N. Saphenous ablation: what are the choices, laser or RF energy. Semin Vasc Surg 2005;18:15-8.

[51] Perrin M. Endoluminal treatment of lower-limb varicose veins by radiofrequency and endovenous laser. Endovascular Today 2007;(Suppl):22-4. 